Service social

\title{
Le travail social dans le Good Lives Model utilisé auprès des auteurs d'agressions sexuelles
}

\section{Michel Dorais}

Volume 63, numéro 1, 2017

Interventions en matière d'agressions sexuelles

URI : https://id.erudit.org/iderudit/1040026ar

DOI : https://doi.org/10.7202/1040026ar

Aller au sommaire du numéro

Éditeur(s)

École de service social de l’Université Laval

ISSN

1708-1734 (numérique)

Découvrir la revue

Citer cet article

Dorais, M. (2017). Le travail social dans le Good Lives Model utilisé auprès des auteurs d'agressions sexuelles. Service social, 63(1), 1-11.

https://doi.org/10.7202/1040026ar
Résumé de l'article

Cet article dresse un parallèle entre certains aspectscaractéristiques du service social contemporain et del'approche du Good Lives Model auprès des auteursd'agressions sexuelles. d'utilisation que vous pouvez consulter en ligne.

https://apropos.erudit.org/fr/usagers/politique-dutilisation/ 


\title{
Le travail social dans le Good Lives Model utilisé auprès des auteurs d'agressions sexuelles
}

DORAIS, Michel

\begin{abstract}
RÉSUMÉ
Cet article dresse un parallèle entre certains aspects caractéristiques du service social contemporain et de l'approche du Good Lives Model auprès des auteurs d'agressions sexuelles.
\end{abstract}

Mots clés : Good Lives Model, service social, résilience, désistance, forces, alternatives.

\begin{abstract}
This article draws a parallel between some aspects of contemporary social service and the Good Lives Model approach to sexual offenders.
\end{abstract}

Keywords: Good Lives Model, social service, resilience, desistance, forces, alternatives.

\section{INTRODUCTION}

Comme entend le montrer ce texte, d'intéressants parallèles peuvent être établis entre le Good Lives Model ${ }^{1}$, tel qu'appliqué aux auteurs d'agressions sexuelles, et plusieurs principes clés du travail social contemporain. Nous retiendrons en particulier la différenciation à faire entre les personnes et leurs conduites, entre leurs symptômes et leurs problèmes de fond, ainsi que l'importance de désistance et de la résilience, de la capacité d'agir des personnes sur leur propre vie, et de la mobilisation de leurs propres forces et de celles de leur milieu de vie. Enfin, le GLM implique une vision stratégique des conduites humaines qui situe ces dernières dans leur finalité et leur contexte. En comprenant les besoins et les motivations que les conduites des auteurs d'agressions sexuelles visent à combler, on est plus à même de trouver avec eux des conduites alternatives, non préjudiciables pour eux-mêmes ou pour autrui, et ainsi d'amenuiser leurs risques de récidives.

Rappelons que le GLM est une approche basée sur le potentiel de changement et de réhabilitation, en particulier des auteurs d'agressions sexuelles ${ }^{2}$. Plus précisément, il reconnaît et soutient leurs forces et mobilise leurs ressources, tant internes qu'externes, plutôt que d'insister sur leurs manques, leurs carences ou leurs troubles de personnalité. En ce sens, le GLM veut amener les auteurs d'agressions sexuelles à satisfaire leurs besoins primaires de façon légitime et socialement acceptable, réduisant ainsi leurs

\footnotetext{
${ }^{1}$ Dans le présent texte, l'abréviation GLM sera utilisée pour désigner le Good Lives Model.

2 Tony Ward \& Theresa A. Gannon, « Rehabilitation, Etiology, and Self-Regulation: The comprehensive good lives model of treatment for sexual offenders », Agression and Violent Behavior, vol. 11, $\mathrm{n}^{\circ}$ 11, 2006, p. 7794; Tony Ward, Ruth E. Mann et Theresa A. Gannon, « The Good Lives Model of Offender Rehabilitation: Clinical implications ", Aggression and Violent Behavior, vol. 12, n 1, 2007, p. 87-107; Geneviève Coco et Serge Corneille, "Quand la justice restaurative rencontre le Good Lives Model de réhabilitation des délinquants sexuels : fondements, articulations et applications », Psychiatrie et violence, vol. 9, n 1, 2009.
} 
risques de récidive. L'accent est mis sur leur faculté d'effectuer des choix différents afin d'éviter la récidive, encourageant ainsi non seulement la cessation de leurs délits ${ }^{3}$ (désistance) mais aussi la reconstruction de leur vie (résilience). Cette perspective est en nette rupture avec le traitement traditionnel des délinquants sexuels, alors que les thérapeutes ne permettaient guère aux individus concernés de prendre leurs propres décisions. C'est aussi en contradiction avec le mythe selon lequel les auteurs d'agressions sexuelles seraient sous l'emprise d'impulsions de nature génétique, hormonale ou psychique irrépressibles, qui feraient en sorte que commettre ces délits serait en quelque sorte «plus fort qu'eux ». Au contraire, le GLM insiste sur la capacité d'agir de l'individu et de sa faculté d'assumer ses décisions et ses choix de vie en tant que personne responsable.

Pour justifier ces prémisses, le GLM postule que les délinquants sexuels ont en général manqué d'opportunités et du soutien nécessaire pour répondre adéquatement à leurs besoins sans porter préjudice à eux-mêmes ou à autrui. Leurs compétences psychosociales et leur développement ont ainsi été freinés. Par exemple, la délinquance sexuelle peut résulter d'une tentative pour soulager un sentiment d'infériorité, d'incompétence, ou encore une crise ou un traumatisme. Les mobiles ou rationnels des auteurs d'agressions sexuelles parlent en somme de besoins qu'ils ont voulu satisfaire. C'est pourquoi le GLM entend permettre aux individus concernés de développer un répertoire de cognitions, de réactions, d'expériences de vie et de projets différents, socialement acceptables et constructifs. En somme, pour le GLM, la délinquance sexuelle est le résultat de stratégies inadaptées, préjudiciables pour soi et autrui, que les auteurs d'abus ont adoptées dans un contexte déterminé afin de satisfaire certains besoins. Le GLM travaille à les amener à élaborer de nouvelles stratégies adaptatives pour répondre à leurs besoins et, pour ce faire, à amenuiser ou éliminer les facteurs de risque présents dans leur vie pour les remplacer par des facteurs de protection.

Quoique non exclusifs, les principes clés du travail social qui se retrouvent au cœur du GLM sont pluriels, comme on le verra. Les passer brièvement en revue, un a un, nous permettra de souligner à la fois leur spécificité, leur pertinence, voire leur originalité dans l'intervention auprès des auteurs d'agressions sexuelles.

\section{La personne n'est pas son comportement}

Plus un acte heurte nos valeurs, plus il est difficile de dissocier la personne qui l'a posé de son comportement. Dans son ouvrage devenu un classique, Délinquants, pourquoi ?, le criminologue québécois Maurice Cusson conçoit le délit comme un comportement orienté vers des résultats, doté de sa rationalité propre, tenant compte des opportunités qui s'offrent au contrevenant dans un contexte donné et des réactions escomptées de la part d'autrui (par exemple, les risques, conflits ou sanctions possibles). Les finalités visées par un acte dit délinquant peuvent donc être très diverses : elles vont du positif au négatif. C'est pourquoi l'intervenant doit se concentrer sur ce que les contrevenants croient et font plutôt que sur ce qu'ils sont, ou seraient.

De surcroît, l'étiquette qu'on accole à un individu (par exemple, « délinquant sexuel ») devient souvent une prophétie auto-réalisatrice. Le psychologue Robert Rosenthal a

${ }^{3}$ D. Richard Laws \& Tony Ward, Desistance from Sex Offending, New York, Guilford Press, 2011. 
appelé ce procédé l'effet Golem, lequel se produit quand l'étiquetage conduit à sousestimer les capacités d'une personne. On a alors tendance à évaluer les gens selon les attentes et les préjugés entretenus à leur sujet, et non selon leurs réelles capacités, limitant ainsi l'éventail des interventions possibles à leur égard.

Les sociologues Erving Goffman et Howard Becker ont développé ce que l'on a appelé la théorie de l'étiquetage ${ }^{4}$. On pourrait la résumer de la façon suivante : devient déviant un comportement que les gens désignent comme tel, et déviante une personne qui a posé ce comportement et à qui on accole dès lors cette étiquette. Une des conséquences du processus d'étiquetage est de réduire l'individu à certains de ses attributs ou à certains de ses actes, qui en viennent à recouvrir l'ensemble de sa personne. Ainsi, sachant que leurs auteurs ont souvent débuté leurs méfaits à l'adolescence, combien d'abus sexuels faut-il qu'ils commettent pour devenir des agresseurs, et plus rien d'autre que cela ?

La stigmatisation sociale permanente des déviants emprunte, en fait, deux voies et génère deux types de limitations : celle qui provient du regard des autres et celle qui provient du regard porté par la personne sur elle-même. L'étiquette peut sembler pratique parce qu'elle procure une illusion de compréhension : on a nommé le problème et trouvé son responsable. Ce procédé expéditif réduit pourtant notre disposition à voir le potentiel de changement des gens. Plus encore, l'étiquette incite la personne à s'y conformer, et par conséquent à maintenir ou même aggraver sa situation en répétant les mêmes conduites problématiques.

La pensée essentialiste remonte aux débuts de la criminologie, alors qu'un expert en médecine légale comme Cesare Lombroso prétendait que ses recherches sur l'anatomie démontraient que l'on naissait criminel. Même dans une version atténuée, qui affirme qu'on ne naît pas forcément criminel mais qu'on le devient très tôt à cause de prédispositions génétiques, hormonales ou autres, la logique essentialiste contribue non seulement à étiqueter les individus, mais aussi à faire en sorte que cette étiquette leur colle durablement à la peau, puisqu'ils ne peuvent précisément pas changer de peau. Or, adhérer à une vision essentialiste est nettement contre-productif pour un intervenant psychosocial parce que cela signifie qu'il ne croit guère au changement chez des individus considérés génétiquement programmés : on voit mal comment il pourrait l'encourager.

On appelle « erreur fondamentale d'attribution » le biais d'interprétation faisant en sorte que l'on réduit un individu à ses dispositions présumées. Dans son ouvrage Sommesnous tous des psychologues?, Jacques-Philippe Leyens dénonce le fait «que nous avons une propension à surestimer, dans nos explications, la part qui provient de l'individu - les causes internes, la personnalité - et à sous-estimer celles qui proviennent de la situation - les causes externes, les circonstances ${ }^{5}$ ». Autrement dit, alors que l'explication de tout problème devrait tenir compte du contexte, nous avons tendance à faire reposer sur la personnalité des gens l'ensemble de leurs comportements. Ainsi, nous croyons à tort qu'un acte délinquant ne peut être que le fait d'un délinquant, qu'une agression le fait d'un agresseur récidiviste, etc.

\footnotetext{
${ }^{4}$ Howard Becker, Outsiders, Paris, Métailié, 1985 ; Erving Goffman, Asiles, Paris, Minuit, 1968, et Stigmates, Paris, Minuit, 1975.

5 Jacques-Philippe Leyens, Sommes-nous tous des psychologues ? Approche psychosociale des théories implicites de la personnalité, Bruxelles, Mardaga, 1983, p. 98.
} 
Or, les contextes de vie, les circonstances et les motivations qui modulent les actions et réactions des gens sont susceptibles d'évoluer. Ce qui peut les amener à changer. La psychologie de tout individu est, après tout, le résultat de son histoire de vie, ce qui inclut le rapport à soi, à ses pairs et ses proches, à sa communauté et aux événements clés qu'il traversera. Si son passé oriente la conduite d'une personne, il ne l'emprisonne pas : l'avenir est une aventure ouverte sur de nouvelles expériences, de nouveaux apprentissages, de nouvelles conduites. Le GLM met précisément le focus sur cette ouverture, qui rend le changement possible à travers le développement de nouveaux scripts de vie ${ }^{6}$.

\section{Le symptôme n'est pas le problème}

Départager ce qui est de l'ordre de symptômes ou de problèmes de fond est un exercice difficile. Pourtant, sans cette discipline de l'esprit, on risque de travailler en pure perte ou même d'empirer le problème en ne traitant pas ses racines. II n'y a pas d'économie à faire sur ce plan : c'est en écoutant longuement l'histoire de vie des gens puis en élaborant des hypothèses sur les origines des symptômes constatés que l'on va pouvoir remonter à leur source, c'est-à-dire aux problèmes dont ces symptômes sont les manifestations. On découvre, par exemple, que les premières agressions sexuelles commises l'ont généralement été dans le cadre de contextes précis, en réponse à des circonstances et des besoins très spécifiques. La passage à l'acte délinquant est souvent une stratégie plus ou moins consciente grâce à laquelle la personne croit pouvoir soulager une crise, une frustration ou un traumatisme ${ }^{7}$. La seule façon de le comprendre est de remonter du symptôme jusqu'à ce problème d'origine.

Le mot "symptôme », rappelons-le, vient du monde médical. Médecin de formation, Freud fut à sa façon un promoteur de l'idée qu'un problème de fond se cache souvent derrière un symptôme, dont il importe de trouver non seulement l'origine mais aussi la signification. En se permettant d'aller toujours par-delà les apparences, en partant du symptôme pour retracer le problème, Freud allait beaucoup influencer l'intervention thérapeutique (cela, bien au-delà de la mouvance psychanalytique).

Une précision s'impose néanmoins quant à la différence qu'il convient de faire entre les origines et les causes d'un problème. En sciences sociales, il serait bien présomptueux de parler de relation de cause à effet, a fortiori si on le fait au singulier : l'histoire de vie des gens constitue un complexe enchevêtrement d'événements multiples, qui interagissent de surcroît les uns avec les autres. II est toutefois possible d'identifier les origines d'un problème, c'est-à-dire le contexte personnel, familial ou social dans lequel ce dernier est apparu puis s'est développé. Comprendre ce contexte permet au moins d'identifier qui est concerné par le problème, qui y contribue, et de quelle façon. Ces informations permettront d'élaborer des hypothèses sur la façon dont se passent les choses et sur les moyens d'en changer le cours dans le futur.

Le symptôme d'un problème constitue souvent une "solution » qui, dans les faits, a empêché tout changement, fût-ce en cristallisant une réaction inappropriée. Par exemple, une victime d'abus sexuels qui croit que l'unique solution pour solutionner son malaise et

${ }^{6}$ S. Maruna, Making Good: How Ex-Convicts Reform and Rebuilt their Lives, Washington, APA, 2001.

${ }^{7}$ Michel Dorais, La mémoire du désir, Montréal, Typo, 2004. 
son traumatisme c'est de renverser les rôles en commettant elle-même des violences aggrave sa situation en essayant de la régler. Son symptôme devient alors aussi un problème pour autrui (cela dit, toutes les victimes ne seront pas tentées de renverser les rôles, il importe de le souligner: plusieurs autres stratégies adaptatives existent, en particulier chez les garçons et les hommes ${ }^{8}$ ).

\section{Les conduites humaines sont stratégiques}

Même les individus qui ont commis les pires crimes ont toujours, de leur point de vue, de «bonnes » explications pour tenter de justifier, voire de légitimer, leurs agissements. C'est pourquoi il convient de comprendre les mobiles, les logiques et les circonstances qui se profilent derrière leur conduite. Chercher à comprendre et à expliquer un comportement ne saurait signifier qu'on l'excuse ou qu'on le banalise, soulignons-le. L'empathie, c'est-à-dire la faculté de se mettre à la place d'autrui, n'est pas synonyme de sympathie, d'autant que cette dernière est souvent non seulement contre-indiquée, mais impossible. Comme l'a écrit avec beaucoup de justesse le psychiatre français Jacques Hochmann: "L'empathie répond à une question : qu'est-ce que l'autre éprouve, que croit-il, que désire-t-il, que pense-t-il ? C'est un geste vers l'autre pour connaître, comprendre ou expliquer son comportement. Si elle nécessite [...] une façon de se mettre à la place occupée par l'autre, elle n'implique pas nécessairement un partage de sentiments ${ }^{9}$. 》

Une dimension essentielle à la compréhension des conduites humaines est qu'elles sont, d'une part, stratégiques, et, d'autre part, contextuelles ou circonstancielles. Comprendre les motivations ou les finalités des actes qu'on veut prévenir exige de les contextualiser, de comprendre le raisonnement et les circonstances qui les ont entourées. Surtout utilisée en sociologie des organisations, la perspective stratégique offre l'avantage de toujours mettre l'accent sur ce que font les gens et non pas sur ce qu'ils sont ${ }^{10}$. C'est dans la mesure où les résultats aujourd'hui obtenus ne coïncident pas, ou plus, avec ses objectifs ou ses valeurs que la personne sera encline à réexaminer sa conduite et à trouver d'autres stratégies pour arriver à ses fins, ou encore à changer sa façon de voir. Autrement dit, tant qu'un comportement, fût-il délinquant, donne les résultats escomptés, il y a de bonnes chances qu'il perdure. C'est souvent la prise de conscience du décalage entre les fins visées (par exemple, la réponse à des besoins estimés légitimes) et les résultats réellement atteints (par exemple, les conséquences néfastes pour soi et autrui) qui va amener la personne à questionner puis à modifier son comportement.

Toute conduite humaine entraîne des gains et des pertes : c'est ce qui amène les individus non seulement à adopter certains comportements mais aussi à les changer quand ils s'avèrent perdants ou destructeurs. Car il y a en général plusieurs façons de répondre aux mêmes besoins. Comme le suggère le GLM, le travail des intervenants auprès d'auteurs d'actes répréhensibles consiste justement à dégager ces alternatives et à encourager l'adoption de celles qui apparaissent les plus viables pour les personnes concernées et pour leur environnement. En comprenant les besoins et les finalités que les conduites sexuellement délinquantes visent à combler (donc leur motivation, leur

\footnotetext{
${ }^{8}$ Michel Dorais, Ça arrive aussi aux garçons, Montréal, Typo, 2008, et Paris, Payot, 2013.

9 Jacques Hochmann, Une histoire de l'empathie, Paris, Odile Jacob, 2012, p. 173.

${ }_{10}$ Michel Crozier et Erhard Friedberg, L'acteur et le système, Paris, Seuil, 1977.
} 
rationalité et leur contexte), on est plus à même de chercher et de trouver des conduites alternatives, non préjudiciables et même constructives.

Faire l'inventaire des gains et des pertes que provoquent les conduites proscrites peut aider à les remettre en question. Le tableau suivant (fruit d'un exercice conceptuel réalisé en classe avec mes étudiants, que je remercie), donne un bon aperçu de la logique de l'approche stratégique et des portes qu'elle ouvre sur le plan de l'intervention.

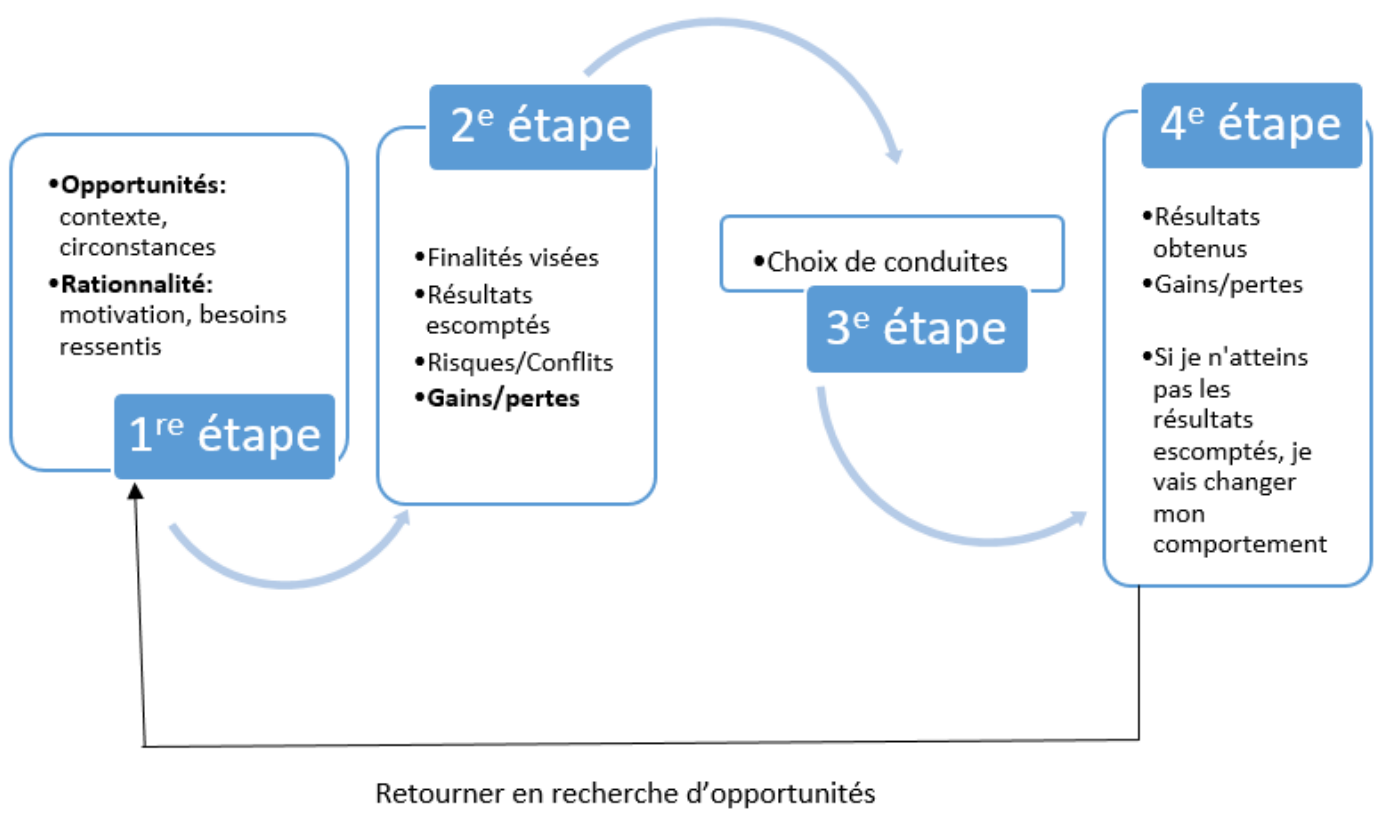

\section{Soutenir la désistance, la résilience et la capacité d'agir}

II y a des moments de grâce, exceptionnels dans tous les sens du terme, durant lesquels même les pires problèmes éprouvés se trouvent en quelque sorte mis entre parenthèses. Comprendre dans quel contexte cela se produit est essentiel. Car ce qui, dans l'existence d'une personne, résiste à ses problèmes est un bel indice que ces derniers peuvent être amenuisés ou résolus.

Beaucoup d'approches thérapeutiques se focalisent sur les failles ou les carences des gens. Cela fait en sorte que beaucoup d'intervenants psychosociaux ont plus ou moins consciemment tendance à ignorer ou à sous-estimer les capacités et les habiletés de leurs clientèles, alors même que ces aptitudes et ces forces peuvent aider ces dernières à s'en sortir. La faculté de s'extirper de ses problèmes malgré des circonstances adverses s'appelle la résilience. Boris Cyrulnik proposait dans un de ses tout premiers ouvrages une définition un peu plus élaborée : " la capacité à réussir à vivre et à se développer positivement, de manière socialement acceptable, en dépit du stress ou d'une adversité qui comportent normalement le risque grave d'une issue négative ${ }^{11}$ ". Si l'on croit au changement, mettre l'accent sur le potentiel des gens, donc sur leur résilience, est incontournable. Même s'il est vrai que la notion de résilience a bien davantage été

${ }^{11}$ B. Cyrulnik, Un merveilleux malheur, Paris, Odile Jacob,1999, p. 10. 
développée en référence à des personnes qui furent victimisées, et très peu dans le cas de personnes qui ont commis des crimes (encore qu'une condition n'exclue pas l'autre).

Chez les auteurs d'actes criminels, le concept de résilience cher au travail social peut aussi être mis en continuité à celui désistance, surtout utilisé en criminologie. Définie comme l'abandon, graduel ou subit, de certaines conduites, la désistance est aussi un résultat qui est le fruit d'un processus ${ }^{12}$. Les concepts de désistance et de résilience sont certes différents, mais la résilience s'inscrit dans le prolongement de la désistance et en soutien de cette dernière : car cesser de faire quelque chose ne permet pas de trouver réponse aux besoins qui étaient comblés par ladite activité. Le GLM met d'ailleurs l'accent sur les facteurs internes (valeurs et développement de compétences, par exemple) et externes (soutien et nouvelles opportunités sur le plan social, par exemple) qui peuvent soutenir un processus de désistance ${ }^{13}$. Nous sommes ainsi sur la voie de la résilience, qui implique justement un processus de reconstruction de soi, un retour «à la normale» sur le plan de l'équilibre personnel et du fonctionnement social. Désistance et résilience peuvent donc, dans le cheminement qu'elles impliquent, être complémentaires, parfois même se recouper ${ }^{14}$.

La résilience, que l'on pourrait décrire comme un processus de ressaisissement de soi, fait appel à plusieurs types de réactions complémentaires. II y a d'abord une faculté compensatoire ou adaptative devant l'adversité (qu'elle soit provoquée ou subie n'y change pas grand-chose). Cela implique une recherche active d'équilibre chez la personne qui va vouloir contrer ses facteurs de vulnérabilité ou de risque en développant des facteurs de protection appropriés dans son quotidien. La personne résiliente a ainsi une nette propension à se définir par des projets de vie plutôt que par son passé; elle est non seulement ouverte au changement, mais active dans sa recherche de mieux-être. Parler de résilience chez des auteurs d'agressions sexuelles, c'est surtout croire que certains d'entre eux peuvent arriver à retrouver le «bon gars» qu'ils étaient avant les circonstances ou les événements qui les ont amené à commettre leur délit et à en subir les conséquences, parfois traumatisantes pour eux aussi, après coup. D'où leur résilience souhaitée, qui consiste à retrouver leur équilibre malgré tout, après cela.

La résilience opère en fait en deux mouvements : une relative résistance à l'adversité (en fût-on responsable, comme dans le cas des auteurs d'agressions sexuelles) et la reconstruction de soi et de sa vie en anticipant un avenir meilleur. La résilience n'est donc pas un attribut inné, mais une faculté susceptible d'être développée. La résilience postule en somme que des réactions ou des conditions favorables peuvent émerger de toute situation, fût-ce en recadrant cognitivement un événement qui serait en tout autre temps vu comme destructeur. Par exemple, le problème paralysant se transforme en défi stimulant. Les intervenants psychosociaux peuvent ainsi devenir des tuteurs de résilience par le soutien qu'ils apportent.

Presque indissociable d'un processus de résilience, la capacité d'agir se manifeste, quant à elle, par un refus de la fatalité et du statu quo. Cela implique une conscientisation qui

\footnotetext{
12 Murana, op. cit.

13 Laws \& Ward, op. cit.

${ }^{14}$ Claire Fitzpatrick, «What is the Difference between Desistance and Resilience ? Exploring the Relationship between Two Key Concepts», Youth Justice, vol. 11, no. 3, 2011; L. Robertson \& al., «Promoting Desistance and Resilience in Young People Who Offend», Scottish Journal of Crime and Justice Studies, no. 12, 2016.
} 
instaure un rapport différent à soi et aux autres, lequel permet le changement. La capacité d'agir implique par conséquent une action visant à (re)prendre sa destinée en main. Pour ce faire, une réorganisation cognitive voire un savoir alternatif sont dans un premier temps requis afin de redéfinir la situation problème pour en arriver à avoir du pouvoir sur cette dernière.

Les personnes qui ont commis des actes délictuels se présentent souvent comme victimes elles-mêmes des événements. Pour s'ouvrir au changement, elles doivent donc reconnaître leur pouvoir d'agir sur leur propre vie et être en mesure d'avoir un regard critique sur leurs décisions antérieures. Cela implique le développement ou le renforcement de leur empathie, de leur conscience critique, de leur estime de soi, si requis, et de leur faculté d'effectuer des choix améliorant leurs conditions de vie, avec le concours de leur environnement et des ressources qui s'y trouvent, bien entendu.

\section{Tabler sur le potentiel et sur les forces des individus et de leur environnement}

Le passé conditionne, et on ne peut l'effacer. Mais, si lourd soit-il, il n'enchaîne pas. Le présent reste ouvert sur le changement dans la mesure où il permet d'avoir des expériences nouvelles et de développer des habiletés qui ouvriront la voie à des projets de vie porteurs de mieux-être. Tabler sur des projets générateurs d'espoir est souvent la meilleure façon d'amener des individus à se défaire des réflexes qui les condamnent à renouveler leurs conduites préjudiciables du simple fait qu'ils n'en connaissent pas d'autres pour répondre à leurs besoins.

Plusieurs approches dites humanistes valorisent le potentiel des individus. C'est sans doute le modèle axé sur les forces, développé à partir des années 1980 par des professeurs de l'École de service social de l'université du Kansas, sous la direction de Charles Rapp, qui a le mieux systématisé cette perspective ${ }^{15}$. Initialement développée pour les clientèles ayant des problèmes de santé physique ou mentale, cette approche met l'accent sur ce qui fonctionne bien chez la personne et sur ce qu'elle souhaiterait réaliser plutôt que sur ses limitations et sa vie passée. Les concepteurs du modèle axé sur les forces insistent sur le fait que tout individu possède la faculté d'apprendre, de changer et d'agir sur sa propre existence. Pour ce faire, il doit puiser dans ses propres ressources et dans celles de son environnement. Hélas, les symptômes et les problèmes qu'il éprouve lui font parfois perdre de vue cette faculté. Le travail auprès de lui consiste alors à le remettre en contact avec ces forces et à les mobiliser.

Selon la logique de cette approche, l'intervenant psychosocial considère toute personne apte à prendre ses propres décisions, quelle que soit la gravité de ses symptômes ou problèmes. Son rôle est d'amener l'individu à trouver ses propres solutions en mobilisant ses ressources internes et externes. Pour tabler sur ses forces, il importe évidemment de bien cerner les compétences, les talents, les intérêts et les aspirations de chacun. Les ressources de son environnement humain, en particulier de son réseau social, doivent aussi être prises en compte et sollicitées dans toute la mesure du possible.

${ }^{15}$ Charles Rapp, «Theory, Principles and Methods of the Strengths Model of Case Management », dans M. Harris et H.C. Bergman, Case Management for Mentally III Patients, Langhome (PA), Harwood Academic Publishers, 1993 ; L. Carlson, D. McDiarmid \& C. Rapp, The Strenghths Model : Case Management with People Suffering from Severe and Persistent Mental Illness, Instructor's Manual, New York, Oxford University Press, 1998. 
L'impact de leur environnement sur le bien-être des gens n'est pas une donnée nouvelle. L'approche écologique (aussi appelée écosystémique), souvent utilisée en travail social et en éducation ${ }^{16}$, met l'accent sur la relation qui s'établit non seulement entre l'individu et son environnement social mais aussi entre les multiples systèmes qui composent ce dernier (la famille, le milieu d'étude ou de travail, le quartier, etc.). Comme ces systèmes interagissent les uns avec les autres, cela ouvre la porte à des interventions qui dépassent, de loin, le seul niveau psychique ou individuel pour inclure les dimension familiale, sociale et collective de l'intervention sociale. Des interventions individuelles qui peuvent soulager des symptômes sont souvent insuffisantes à elles seules pour régler un problème ou prévenir sa récidive. Le milieu même de vie de la personne doit être mobilisé afin de la soutenir dans les changements qu'elle désire opérer.

\section{La faculté d'autodéternination}

La travailleuse sociale Insoo Kim Berg, une pionnière de la thérapie brève, affirmait que le rôle de l'intervenant est d'aider la personne à trouver ses propres solutions. Elle posait trois balises à ce qui pouvait dès lors être encouragé, ou pas. D'abord, ne jamais tenter de réparer ce qui n'est pas cassé : autrement dit, ne pas toucher à ce qui va bien. Ensuite, si une solution a fonctionné au moins une fois, il vaut la peine de la réessayer encore. En revanche, il ne sert à rien de tenter d'appliquer à nouveau une solution qui n'a jamais fonctionné : cela ne peut que générer du découragement ${ }^{17}$.

Une certaine directivité est nécessaire pour motiver le changement et faire en sorte qu'il advienne. Si l'aidant ne doit pas se percevoir comme un dispensateur de recettes toutes faites, il est néanmoins responsable du processus qui permettra de trouver des solutions. En fait, toutes les études portant sur les facteurs qui contribuent à la réussite d'interventions de nature thérapeutique montrent que quatre éléments s'y retrouvent presque toujours. Ils constituent ce que l'on appelle l'alliance thérapeutique, laquelle constitue un must, y compris en intervention en contexte d'autorité ${ }^{18}$. Elle présente les quatre caractéristiques suivantes:

- la personne aidée et l'intervenant se sont entendus sur des objectifs clairs à atteindre;

- une relation de confiance entre l'intervenant et la personne aidée est établie et maintenue;

- l'autodétermination de la personne, c'est-à-dire le fait qu'elle participe aux décisions qui la concerne, est respectée et encouragée;

- enfin, l'intervenant est en mesure d'expliquer la logique de l'action qu'il prône.

Selon le chercheur et thérapeute Barry L. Duncan et ses collègues, «l'efficacité thérapeutique réside pour l'essentiel dans le ressenti du patient et dans l'utilisation d'une relation améliorant le moral, renforçant les ressources, et apportant de la motivation, avec

\footnotetext{
16 Le pionnier de cette approche est le psychologue américain Urie Bronfenbrenner (1917-2005), dont les deux principaux ouvrages sont: The Ecology of Human Development, Cambridge, Harvard University Press, 1979 et Making Human Beings Human: Bioecological Perspectives on Human Development, Sage, 2005.

17 Insoo Kim Berg et Yvonne Dolan, Récits de solutions, chapitre 7, Québec, Édisem, 2002.

18 M. McMurran, Motivating Offenders to Change, Chichester, Wiley, 2002.
} 
un thérapeute délivrant du soutien et des défis à relever (dans des proportions et à des moments correspondant aux besoins et aux capacités du patient) ${ }^{19}$. Plus l'alliance thérapeutique s'installe tôt, meilleurs sont les résultats, car elle permet de s'entendre sur les buts à atteindre et d'amorcer les tâches à accomplir pour améliorer la situation.

\section{Amenuiser les risques en rehaussant les facteurs de protection}

On peut tenter de jauger les chances de succès d'une intervention psychosociale auprès d'auteurs d'agressions sexuelles, et anticiper les risques encourus, mais on ne peut jamais être assuré de ses résultats. Aucun pronostic ne peut se transformer en prophétie. Un intervenant psychosocial travaille avec des hypothèses de travail et des anticipations, et non pas des certitudes. Seuls les résultats concrets perçus dans la vie des gens montreront si ces hypothèses et pronostics étaient justes, ou pas. La vie étant tissée d'aléas, l'issue même des situations à risque demeure imprévisible puisqu'elle dépend non seulement de la personne et de son entourage, mais de circonstances et de contextes parfois aléatoires. Le risque zéro n'existe pas, il convient de le rappeler.

Le philosophe des sciences Karl Popper a consacré une bonne partie de son œuvre à combattre l'idée que l'on peut tirer des prédictions certaines de l'expérience. Le procédé logique qu'on appelle l'induction, qui consiste à se fier au passé pour prédire l'avenir, n'est jamais fiable à $100 \%$. Au mieux, il sert à établir une plausibilité ou des probabilités. Des études et des expertises peuvent certainement soutenir la réflexion et guider l'action, mais l'exercice du jugement professionnel est toujours requis pour adapter ce que prescrivent les théories, la recherche et même l'expérience.

Popper soulignait que les théories sont des filets destinés à capturer la réalité. Hélas, ces filets sont imparfaits. Dans le domaine des comportements humains, la prédiction est une activité particulièrement hasardeuse. Elle est pourtant prisée : on demande fréquemment à des experts de supputer les risques d'une libération conditionnelle accordée à un détenu ou les chances de réussite d'une réinsertion sociale, par exemple. Il existe bien sûr des statistiques provenant de recherches évaluatives pour aider à la prise de décision. Mais l'honnêteté intellectuelle la plus élémentaire devrait nous inciter à reconnaître qu'il est scientifiquement impossible de nous prononcer avec certitude sur l'avenir d'un individu, ne serait-ce que parce que de nouveaux événements sont à tout moment susceptibles de changer le cours «prévisible» de sa vie.

Si nos observations antérieures permettent de fabriquer des hypothèses et de faire des prédictions circonstanciées, la justesse de celles-ci demeure donc toujours sujette à caution. Ce sont des conjectures. Par exemple, le fait que plusieurs individus ayant déjà commis des agressions sexuelles ne récidivent pas est certainement une bonne nouvelle, mais cela ne nous aide guère à prédire précisément qui le fera, ni dans quel contexte. Une façon de composer avec les risques afin de les minimiser est de réunir des facteurs de protection pour les neutraliser, en autant que faire se peut. Rappelons que les facteurs de risque aggravent la vulnérabilité des personnes et accroissent les dangers auxquels elles sont exposées, alors que les facteurs de protection permettent de tabler sur leurs potentiels, leurs forces et leurs acquis, sans oublier les ressources de leur milieu.

${ }^{19}$ B. Duncan, S. Miller, B. Wampold et M. Hubble, L'essence du changement, Bruxelles, De Boeck, 2012 , p. 16. 
À défaut de pouvoir prédire l'avenir et le succès ultime de nos interventions, nous pouvons nous assurer qu'un filet de sécurité minimisera les facteurs de risques et maximisera les facteurs de protection - à la condition que ce filet tienne. L'incapacité à prévoir l'avenir ne constitue pas une excuse pour nous condamner à l'inaction. C'est seulement un motif supplémentaire pour faire preuve de rigueur et pour prendre les précautions qui s'imposent devant un danger identifié.

Intervenir auprès d'auteurs d'agressions sexuelles dans une perspective de réhabilitation et de réinsertion, c'est savoir prendre des risques, mais des risques calculés, pris en connaissance de cause et de concert non seulement avec les personnes concernées mais aussi avec les ressources du milieu. Avoir foi dans la réhabilitation ou la réinsertion des auteurs d'abus sexuels ne confine pas à la naïveté, encore moins à l'imprévoyance. Au contraire, c'est en étant bien au fait des forces des personnes aidées que nous apprenons à ne pas les outrepasser, provoquant ainsi des échecs qui marqueront des retours en arrière. Aider quelqu'un vers sa réhabilitation ou sa réinsertion, c'est accompagner ses pas, et non pas les faire à sa place, encore moins les précipiter.

\section{En guise de conclusion}

Qu'il soit implicite ou explicite, l'apport de principes clés du service social au GLM est significatif. Car, en définitive, c'est une perspective humaniste qui caractérise ce dernier. Cette vision implique de croire à la faculté de chaque être humain de se sortir de symptômes et de problèmes qui le perturbent grâce à ses ressources personnelles et au soutien de son environnement social. Tout risque étant en grande partie contextuel, le GLM procède d'un sentiment de confiance non pas aveugle mais éclairé, voire balisé par des principes limpides et mobilisateurs ${ }^{20}$ mis au service de la réhabilitation et de la réinsertion.

Comme cadre conceptuel orienté vers l'action, le GLM a presque révolutionné la façon de concevoir les auteurs d'agressions sexuelles et les interventions à leur endroit. Bien qu'il ait emprunté à diverses traditions, il est animé à la fois par un esprit critique et novateur et par le souci de s'appuyer sur des données probantes afin d'éviter tout dogmatisme et de bonifier continûment ses pratiques.

Tel que le soulignaient Laws et Ward ${ }^{21}$, le GLM est soutenu à la fois par des principes éthiques, humanistes et scientifiques : Qu'est-ce qui est dans le meilleur intérêt du client ? Qu'est-ce qui est dans le meilleur intérêt de la communauté? Quelles sont les meilleures pratiques à mettre de l'avant, d'après ce que nous enseignent l'expérience et la recherche? La réponse à ces questions requiert une vigilance intellectuelle et un développement d'expertise qui sont, en principe, au cœur des préoccupations de tout professionnel de la relation d'aide, qu'il soit travailleur social, psychologue, criminologue, psychiatre, ou autre.

Michel Dorais

Professeur titulaire

\footnotetext{
20 L'ensemble des principes clés de la relation d'aide sont développés, beaucoup plus dans le détail, dans mon ouvrage Le métier d'aider, Montréal, VLB éditeur, 2015.

${ }^{21}$ Laws \& Ward, op cit, p. 188 et suivantes.
} 